\title{
Epidural Analgesia Following Component Separation Hernia Repair: Is It Beneficial?
}

\author{
Emily L. Albright, Curtis E. Bower, Daniel L. Davenport, John S. Roth \\ Department of Surgery, University of Kentucky College of Medicine, Lexington, Kentucky \\ Email: s.roth@uky.edu
}

Received April 13 ${ }^{\text {th }}, 2013$; revised May $15^{\text {th }}, 2013$; accepted May 23 ${ }^{\text {rd }}, 2013$

Copyright (c) 2013 Emily L. Albright et al. This is an open access article distributed under the Creative Commons Attribution License, which permits unrestricted use, distribution, and reproduction in any medium, provided the original work is properly cited.

\begin{abstract}
Purpose: Optimal pain management strategies for patients undergoing component separation hernia repair are not defined. Epidural analgesia (EA) has been shown to decrease pulmonary complications and duration of ileus and to improve pain control in other patient populations. In this study we examined outcomes of patients receiving EA after separation of components (SOC). Methods: After obtaining IRB approval, a retrospective review was performed of patients undergoing ventral hernia repair with SOC from January 2006 to October 2010 at the University of Kentucky. Patients were identified from hospital operative records. Pre-operative patient characteristics and operative data were obtained from the medical record. Information was collected relating to use of EA, complications, and length of hospitalization (LOS). Post-operative outcomes were compared between those that had epidurals and those that did not. Results: One hundred seventeen patients were identified that underwent SOC, 34 of whom had EA. These two groups were similar in relation to age, BMI, and co-morbidities. Three patients in the epidural group had complications limiting epidural duration - two with hypotension and one with refractory pruritus. There was no difference in pneumonia, deep vein thrombosis (DVT), wound infection, urinary tract infection (UTI), recurrence, or mortality (Table 1). There was an increase in LOS (6.68 vs. 6.06 days, p < 0.01) in patients with EA. Conclusions: The use of EA results in increased LOS in patients undergoing SOC. EA associated morbidity occurs infrequently. The incidence of post-operative complications is unaffected by EA. Further studies are needed to delineate the benefit of EA in this patient population.
\end{abstract}

Keywords: Hernia Repair; Separation of Components; Pain Management; Length of Stay

\section{Introduction}

Within the last decade, there has been an appreciation for the importance of post-operative pain control. Pain management standards were introduced by the Joint Commission on Accreditation of Healthcare Organizations in 2001 [1]. The ideal post-operative pain control strategy would provide good pain relief, be easy to administer, and have minimal complications.

Ventral hernia repair is a commonly performed general surgery procedure with separation of components used to repair complex hernias. Separation of components involves division of the external oblique to facilitate an autologous tissue repair. This potentially creates large tissue flaps and requires mobilization of myocutaneous layers which can result in significant post-operative pain. Optimal post-operative analgesia has not been defined for patients undergoing ventral hernia repair with separation of components. From our clinical observations and anecdotal experience, it appeared that patients undergo- ing EA had better pain control and were discharged earlier when compared to patients without EA. This study aims to examine the outcome of patients receiving epidural analgesia after separation of components.

\section{Methods}

After obtaining approval from the Institutional Review Board all patients undergoing separation of components and ventral hernia repair from Patients were identified from the hospital operative records. Patients were excluded if they had separation of components in association with closure of a staged abdominal operation. Those patients undergoing operation by the departments of pediatric surgery, plastic surgery, transplant surgery, and gynecologic surgery were also excluded as their clinic charts were not available. Data were extracted from the medical record including age, gender, body mass index (BMI), co-morbidities, previous ventral hernia repairs, and presence of infection at time of surgery. Operative 
Table 1. Pre-operative and operative characteristics.

\begin{tabular}{cccc}
\hline & Epidural & No Epidural & $\mathrm{p}$ \\
\hline Number of Patients & 34 & 83 & 0.29 \\
Age (mean) & 51.9 & 49.5 & 0.05 \\
Gender (M:F) & $21: 13$ & $34: 49$ & \\
Co-morbidities & & & 0.13 \\
DM & 7 & 30 & 0.54 \\
HTN, CAD, CHF & 19 & 52 & 0.80 \\
COPD/Asthma & 6 & 17 & 0.29 \\
Tobacco Use & 17 & 28 & 0.01 \\
ASA > 2 & 11 & 49 & 0.49 \\
BMI (mean) & 33.5 & 34.9 & 0.89 \\
Number Prior Repairs (mean) & 0.94 & 0.94 & 0.73 \\
Mesh Size cm ${ }^{2}$ (mean) & 337 & 355 & \\
\hline
\end{tabular}

data included American Society of Anesthesiology (ASA) class, and size of mesh (if used). The use of epidural analgesia, duration of epidural use, and any associated epidural complications were recorded. Outcomes examined included post-operative complications, hospital length of stay, mortality and hernia recurrence.

All epidurals were placed pre-operatively by the anesthesia and pain management service. The decision for EA was based on patient preference, body habitus, medical history, as well as anesthesiology team preference. The type of anesthesia used for EA was a mix of narcotics and local anesthetics. EA patients were placed on standard intravenous and patient controlled anesthesia and transitioned to oral narcotic pain medication when they began to tolerate a diet.

We compared patient characteristics, operative factors, and post-operative outcomes between those that received epidural analgesia and those that did not using a t-test for approximately normal continuous variables, Mann Whitney $U$ test for length of stay and prior repairs and a Fischer exact test for dichotomous variables. A p value of less than 0.05 was considered significant. Statistical analysis was performed with SPSS ${ }^{\mathrm{TM}}$ v.20 (IBM Corp, New York, NY).

\section{Results}

One hundred seventeen patients were identified during the study period that underwent ventral hernia repair with separation of components. Of these, 34 received epidural analgesia for post-operative pain control. Males more frequently received epidurals comprising $62 \%$ of the epidural group vs. $41 \%$ in the non-epidural group (p = $05)$. There were no significant differences in relationship to age, the presence of co-morbidities, BMI, number of prior repairs, implantation of mesh, and mesh size be- tween the two groups. Mean defect size in the epidural group was $275.9 \mathrm{~cm}^{2}$ and $119.2 \mathrm{~cm}^{2}$ in the non-epidural group ( $<0.001$ ). ASA score was lower in the EA group (ASA > 2: EA 32\% and non-EA 59\% p < 0.01). Patient groups were otherwise similar (Table 1). Of the $34 \mathrm{pa}-$ tients that had epidural analgesia, three patients had complications which limited epidural duration, two patients had hypotension, and one patient developed refractory pruritus. No patients had infectious complications associated with epidural use.

There was no difference in pneumonia, deep vein thrombosis, urinary tract infection, wound infection, hernia recurrence, or mortality in those with epidural analgesia and those without. There was an increase in hospital length of stay associated with epidural analgesia (6.68 days vs. 6.06 days, $\mathrm{p}<0.01$ ) (Table 2).

\section{Discussion}

Epidural analgesia has been shown to provide superior pain control compared to parenteral opioids in the postoperative period when examining a variety of surgical

Table 2. Post-operative outcome.

\begin{tabular}{cccc}
\hline & Epidural & No Epidural & $\mathrm{p}$ \\
\hline Number of Patients & 34 & 83 & \\
LOS (mean days) & 6.68 & 6.06 & $<0.01$ \\
Pneumonia & 4 & 9 & 1.00 \\
DVT/PE & 0 & 0 & $\mathrm{~N} / \mathrm{C}$ \\
UTI & 3 & 1 & 0.07 \\
Wound Infection & 7 & 30 & 0.13 \\
Recurrence & 4 & 13 & 0.78 \\
Mortality & 0 & 2 & 1.00 \\
\hline
\end{tabular}


procedures [2]. Epidural analgesia not only provides superior analgesia at rest but also during movement or coughing [2,3]. Significant pain can occur post-operatively in patients undergoing ventral hernia repair, especially when separation of components is added. In longterm follow-up after ventral hernia repair, thirty-six percent of patients undergoing primary suture repair and twenty percent of patients undergoing mesh repair re ported abdominal pain in the previous month with a median follow-up of 75 and 81 months respectively [4].

The use of general anesthesia during abdominal surgery can have multiple effects on the pulmonary system including atelectasis, hypoxia, and infection. It has been theorized that epidural analgesia may reduce pulmonary complications due to improved analgesia and resulting increased mobility, deep inspiration, and coughing postoperatively. A meta-analysis looking at pulmonary complications found a decrease in the odds of pneumonia associated with epidural analgesia [5]. This study found that epidural analgesia was significantly more efficacious in preventing pneumonia in trials where controls did not receive patient-controlled analgesia (PCA) compared to trials where controls did have PCA. This meta-analysis also found that epidural analgesia was associated with a decreased odds of prolonged ( $>24$ hours) ventilation and re-intubation. Another meta-analysis had conflicting results regarding pulmonary complications and epidural use. They found a better preservation of the FVC (forced vital capacity) at twenty-four hours post-operatively and a faster time to extubation but no difference in pulmonary complications (pneumonia and atelectasis) [3].

In our patient population, there was no difference in the incidence of postoperative pneumonia between the two groups. However, we were unable to determine length of supplemental oxygen required, ability to use an incentive spirometer, and maximal lung capacity. The wide dissection required in these operations with incision to the xyphoid process is likely to result in some respiratory embarrassment as a result of pain. In one series, pneumonia was seen in 3.9\% (5/128), respiratory failure requiring mechanical ventilation in $3.9 \%$ (5/128), and pleural effusions in $2.3 \%$ (3/128) [6]. The only reliable pulmonary endpoint that was able to be determined was documented pneumonia. Although the difference in pneumonia was not different, our study size is underpowered to detect any significant differences based upon the historical likelihood of developing pneumonia.

The mean length of stay for patients undergoing ventral hernia repair and separation of components varies widely in the literature from 3.4 days to 11 days [7-9]. Clearly, multiple factors play into length of stay including underlying co-morbidities, perioperative complications, and ability to return to a preoperative functional state. In our study we identified an increased length of stay associated with epidural analgesia, contrary to our original hypothesis. Although surprising, the hospital stay of those who received EA seems to be more uneventful. Further studies evaluating metrics of patient comfort as well as specific measures of pulmonary toilet would be beneficial in determining the benefits of EA. Tradeoffs may exist between benefits and risks when utilizing EA. In patients undergoing colorectal surgery there was no reduction in length of stay despite a significantly decreased duration of ileus [10].

This study was conceived based upon the anecdotal observation that patients receiving epidural analgesia postoperatively recovered more quickly. However, there may be inherent selection biases that account for the differences in length of stay in the group receiving epidural anesthesia. Although all patients are recommended to have an epidural catheter preoperatively by the surgical team, both the patient and anesthesiologist are involved in the decision to place the epidural catheter. Anatomic abnormalities and co-morbid conditions influencing the decision to receive epidural analgesia may have impacted hospital length of stay.

A variety of complications can occur with the use of epidural analgesia. While there is a decreased risk of nausea and sedation when compared with intravenous narcotics, there is a higher risk of pruritus, urinary retention, and motor block [10,11]. Epidural analgesia has also been shown to significantly increase the odds of intraoperative hypotension [5]. The use of epidural analgesia also has risks associated with the placement of a catheter including spinal hematoma, cauda equina syndrome, purulent meningitis, and epidural abscess. Fortunately these complications are rare and none occurred in our patient population.

In our patient population there were three patients that had complications associated with epidural use (8.8\%). All three were self-limited complications but resulted in discontinuation of epidural use. All were included in the epidural group for analysis but received traditional analgesia consisting of both intra-venous and oral opioids once the epidural was removed. It is difficult to determine whether the patients benefitted or not from the epidural analgesia or if their outcome was altered by the early discontinuation. However, their inclusion in the epidural group based on an intent to treat as it can be anticipated that a small percentage of patients receiving epidural analgesia will fail treatment.

Interestingly, our EA group was found to have a significantly larger mean defect size than those in the nonEA group (275.9 $\mathrm{cm}^{2}$ and $\left.119.2 \mathrm{~cm}^{2}, \mathrm{p}<0.001\right)$. This tendency may indicate the underlying clinical bias to offer patients with larger hernia defects EA. Although intuitive that larger hernia defects result in greater dissection and may result in greater postoperative discom- 
fort, there is no evidence that hernia size directly impacts postoperative length of stay. However, this group also was noted to have a lower overall ASA score. It is difficult to discern whether patients with higher ASA scores were discouraged from receiving epidural analgesia, were not candidates due to anatomic or physiologic reasons, or simply preferred to not receive this form of pain control. This undoubtedly warrants further investigation as predicting prolonged hospital stays following hernia repair would help target therapies at improving outcomes.

This study has several limitations. It represents the use of epidural analgesia in a select patient population, namely those undergoing separation of components and ventral hernia repair. As such we have a relatively small sample size which limits the conclusions. Over the course of five years we were only able to identify 34 patients that had epidural analgesia at the time of component separation hernia repair. Generally, the component separation procedure patients are more complicated, require greater resources, and longer hospital stays than patients undergoing more straightforward hernia repairs. As a result, we do not feel the results of this study would be generalizable to all hernia patients.

The retrospective nature of this study further limits this study. Information regarding patient satisfaction, pain scores, or patient mobility which would likely be studies in a prospective analysis was not available. The anesthesia pain management team was responsible for managing the epidural analgesia postoperatively but there was not a standardized protocol for medication dosing. Additionally, postoperative care and pain control was not strictly standardized and could allow for some bias. Prospective studies controlling for these variable are planned.

While not statistically significant there were three patients with urinary tract infections in the epidural group compared to one in the group not receiving EA. This may be related to patients having a urinary catheter for a longer duration in the epidural group. EA patients were treated with a foley catheter until six hours following epidural removal while urinary catheters were removed in patients without EA. Following colorectal surgery epidural analgesia has been demonstrated to be associated with an increased risk of urinary retention [10].

Clearly, optimal pain management is a goal following every procedure. Our study suggests that the benefits of epidural analgesia may be mitigated by prolonging hospital stay. Is is dependent on multiple factors and must take into account the patient, the procedure, available resources and overall pain management. Epidural analgesia represents one strategy for patients undergoing separation of component and ventral hernia repair. Further prospective studies are needed to delineate the bene- fits in this patient population. Short-term outcomes are clearly of interest, including pain control, mobility, postoperative complications, and patient satisfaction. However, long term outcomes such as alterations in the incidence of chronic pain following ventral hernia repair need to be evaluated.

\section{Conclusion}

Epidural analgesia following ventral hernia repair with component separation is performed frequently among patients with co-morbid conditions. Postoperative outcomes are similar between patients receiving epidural analgesia and those treated with parenteral narcotics in the early postoperative period, but hospital length of stay is increased in those receiving epidural analgesia. Further prospective studies evaluating the impact of epidural analgesia on patient satisfaction, pulmonary complications, and pain control are planned.

\section{REFERENCES}

[1] The Joint Commission, "Facts about Pain Management," 2011.

www.jointcommission.org/assets/1/18/Pain_Management .pdf

[2] B. M. Block, S. S. Liu, A. J. Rowlingson, J. A. Cowan and C. L. Wu, "Efficacy of Postoperative Epidural Analgesia: A Meta-Analysis," The Journal of the American Medical Association, Vol. 290, No. 18, 2003, pp. 24552463. doi:10.1001/jama.290.18.2455

[3] J. Guay, "The Benefits of Adding Epidural Analgesia to General Anesthesia,” Journal of Anesthesia, Vol. 20, No. 4, 2006, pp. 335-340. doi:10.1007/s00540-006-0423-8

[4] J. W. Burger, R. W. Luijendijk, W. C. Hop, J. A. Halm, E. G. Verdaasdonk and J. Jeekel, "Long-Term Follow-Up of a Randomized Controlled Trial Comparing Suture versus Mesh Repair of Incisional Hernia,” Annals of Surgery, Vol. 204, No. 4, 2004, pp. 578-585.

[5] D. M. Popping, N. Elia, E. Marret, C. Remy and M. R. Tranier, "Protective Effects of Epidural Analgesia on Pulmonary Complications after Abdominal and Thoracic Surgery: A Meta-Analysis,” Archives of Surgery, Vol. 145, No. 10, 2008, pp. 990-999.

doi:10.1001/archsurg.143.10.990

[6] J. M. Clarke, "Incisional Hernia Repair by Fascial Component Separation: Results in 128 Cases and Evolution of Technique," The American Journal of Surgery, Vol. 200, No. 1, 2010, pp. 2-8. doi:10.1016/j.amjsurg.2009.07.029

[7] K. C. Harth and M. J. Rosen, "Endoscopic versus Open Component Separation in Complex Abdominal Wall Reconstruction," The American Journal of Surgery, Vol. 199, No. 3, 2010, pp. 342-347.

doi:10.1016/j.amjsurg.2009.09.015

[8] M. Moore, T. Bax, M. MacFarlane and M. S. McNevin, "Outcomes of the Fascial Component Separation Technique with Synthetic Mesh Reinforcement for Repair of Complex Ventral Incisional Hernias in the Morbidly 
Obese," The American Journal of Surgery, Vol. 195, No. 5, 2008, pp. 575-579. doi:10.1016/j.amjsurg.2008.01.010

[9] M. J. Rosen, J. Jin, M. F. McGee, C. Williams, J. Marks and J. L. Ponsky, "Laparoscopic Component Separation in the Single-Stage Treatment of Infected Abdominal Wall Prosthetic Removal," Hernia, Vol. 11, No. 5, 2007, pp. 435-440. doi:10.1007/s10029-007-0255-y

[10] E. Marret, C. Remy and F. Bonnet, Postoperative Pain
Forum Group, "Meta-Analysis of Epidural Analgesia versus Parenteral Opioid Analgesia after Colorectal Surgery,” British Journal of Surgery, Vol. 94, No. 6, 2007, pp. 665-673. doi:10.1002/bjs.5825

[11] S. S. Liu and C. L. Wu, "The Effect of Analgesic Technique on Postoperative Patient-Reported Outcomes Including Analgesia: A Systematic Review," Pain Medicine, Vol. 105, No. 3, 2007, pp. 789-808. 